\title{
Supporting Improved Maternity Care by Midwives: Design Opportunities and Lessons Learned
}

\author{
Abdullah Al Mahmud and David V. Keyson \\ ID-StudioLab, Industrial Design Engineering \\ Delft University of Technology, 2628 CE Delft, The Netherlands \\ \{a.almahmud, d.keyson\} atudelft.nl
}

\begin{abstract}
In this paper we describe a study about the role of Information Technology (IT) on the quality of maternity care in the midwifery centres in the Netherlands. We conducted an interview study with midwives in the Netherlands. The objective was to understand the current situation, challenges and design opportunities that could help to provided improved healthcare. The results of the interview study show that the current prenatal care system suffer from some challenges such as proper IT support, lack of IT training for the midwives, lack of integrity between different software systems used in the midwifery centres and hospitals and attitude of the pregnant mothers. Based on our findings we provide some recommendations and design implications to support improved care provided by the midwives.
\end{abstract}

Keywords: Prenatal care, midwives, Information Technology (IT), maternal health, Human-Computer Interaction (HCI).

\section{Introduction and Background}

A key aspect to improve maternal health is to make use of the role of skilled health professionals, specifically midwives. Women around the world depend on the expertise of midwives for both prenatal and neonatal care. In the Netherlands, midwives are independent medical practitioners who work either in a midwifery practice centre or in a hospital. Approximately $78 \%$ of pregnant women start their maternity care with a primary care setting where $44 \%$ start labour in primary care, and finally $33 \%$ of women give birth under supervision of a primary care midwife [10]. Midwives are trained to provide maternity care to women whose pregnancy and childbirth are uncomplicated including antenatal, intrapartum and postnatal care to mother and child [1]. The first appointment at the midwifery practice usually takes place around the 8th week of pregnancy. On average one pregnant woman visits midwives 10-13 times over 9 months. Due to the frequent visits throughout pregnancy and because of their expertise, midwives are treated as dependable providers of pregnancy related health education and advice for pregnant women.

Maternity care in the Netherlands is known for its high percentage of home births and for the independent situation of midwives [13, 16]. In the Netherlands about 175,000 births occur annually [2]. Obstetric care in the Netherlands is provided by 
primary caregivers: midwives or general practitioners (GPs), and by specialist obstetricians. Women with low-risk pregnancies receive antenatal care from midwives. When they remain low risk throughout pregnancy they are free to choose to give birth either at home or in hospital. Their midwife (or GP) will support them in their choice and attend the birth in the chosen place. Referral to specialist care only happens in case of complications [1].

Each year worldwide 529000 maternal death occur [3]. The target set for MDG-5 is a 75\% reduction of maternal mortality ratio between 1990 and 2015. Developing countries are mainly struggling to achieve the Millennium Development Goal (MDG). There are three main approaches of safe motherhood strategies which are developed based on different stages in a women's reproductive cycle. The stages are categorized as pregnancy, antenatal, delivery and postpartum period [7]. Antenatal care consists of a number of interventions administered to women during pregnancy, containing screening tests, immunizations, and treatment for recognized complications. It has been established that the detection of early signs or threat factors for morbidity and mortality can be spotted and effective interventions are possible [12]. The main aim of antenatal care is to produce a healthy mother and baby. Midwives have a significant impact on the wellbeing of women and new born babies.

Our aim is to identify needs and desires of midwives about the tools they need to deliver the best possible care for both mothers and infants. We need to get a clear picture of maternity care and their providers in developed countries though it is assumed that developed countries have better maternal care. There has been ample literature on midwives and maternal care in low resource environments for example see $[7,12,15]$. Furthermore, Human-Computer Interaction (HCI) community is working with midwives to design better systems such as improved ultrasound [6]. There are few studies which explored role of computer and Information Technology for midwives in developed countries. For example, one study conducted in Australia to understand the use of Internet among midwives [9]. It was revealed that midwives had insufficient Internet competence. We are not aware of any study that investigates workflow of midwives and the role of IT. Therefore, we explore maternity care issues and challenges in the Netherlands with respect to use of Information Technology (IT) in the midwifery centres. To better comprehend and support the workflows of midwives we conducted an interview study to discover the needs of midwives, and to develop technologies that will help them to better serve their patients. The specific objectives of the study are: a) identifying the role of IT and point-of-care testing (POC) devices b) identifying the purposes of Information Technology (IT) and POC for midwives, c) understanding the barriers to using IT and POC devices in the midwifery centres, d) recommendations to overcome these barriers to improve maternal care.

\section{Interview Study and Results}

In total 15 midwives participated in a semi-structured interview. They were contacted via the Royal Dutch Organization of Midwives (KNOV) [4]. The selection criteria were: the midwives should have active participation in midwifery centres and they should have minimum 5 years of working experience. The participation was voluntary. The preliminary questions for the interviews were a) key issues related to 
providing basic prenatal care, b) managing information to support prenatal care, c) prenatal visits and quality of care, d) barriers and facilitators to the prenatal care and role of Information Technology. Each interview session was one hour long. Interview data were audio recorded and later transcribed. The data were thematically analysed by the first author and one external researcher and the qualitative data analysis method described in [14] was followed. The key themes that emerged from the interviews are explained below:

Protocols for Midwives. Generally midwives (MW) follow written protocols in the midwifery centres; however midwives with several years of experience do not need those very often. One midwife (MW3) mentioned, "some are written and some are in my head, it is in my head as I am experienced and for new colleagues it is on paper as well. We have several booklets in different languages.'

Protocols are written by the Dutch organization of midwives (KNOV). It is a manual to inform how a usual prenatal care should be carried out. However, midwives are not forced to do that. One midwife (MW1) mentioned, 'You have your own responsibilities and you can decide based on your experiences and judgment when to see the patient in two week or four weeks. However, the rules are means and they are not aims'. There are several protocols that are suitable for midwifery centres and regular hospitals where prenatal care is given as well. One midwife (MW9) mentioned, 'we work together with hospitals and we need to know the protocols of the hospital. We have not put the protocols on paper; they change a lot so it is in digital format as PDF files'.

Patients and Communication. Midwives use both paper and electronic forms to manage patient information. During or before the first visit a prenatal registration form is given to the pregnant women and later those are used to keep online record with the help of the software systems that midwives use. One midwife (MW7) mentioned, "when I take a blood sample for testing, I fill out a form, the results come back online and I decide if this is ok or not. If it is not $O K$ the patients are referred. If I have to go an urgent consultation I call the obstetrician". Furthermore, electronic agenda are quite often used to manage and provide care which helps them to work efficiently. Another midwife (MW5) mentioned, "we have agenda per location and we also travel in the 5 locations to provide care so that pregnant women can easily reach. The agenda helps us to keep track of all the appointments".

During the first prenatal visit midwives collect the history of the patients and keep in their software systems. They also provide information to pregnant women about their practice and give lot of information about pregnancy such as diet, exercise and follow up visits Therefore, it is important that the provided information given to pregnant women are easy to remember and manage during the 9 months.

Managing Information and Supporting Prenatal Care. We identified that all the midwives complained about the software systems that they use to manage patient information. There are numerous major advantages of the software systems as well as reported by the midwives. The key advantages are a) patient summary information such as pregnancy history, disease history, family history are easily available, b) an agenda in the software system to manage appointments with the pregnant women. 
The other things are, a) counselling information: smoking and drinking, b) provides summary of the health situation of the pregnant women based on self-reported data. One midwife (MW12) mentioned, "If my colleague fills it I can just access the data. I always read the whole journal and it helps me to get an overall impression about a patient's condition". Therefore, the software systems help to maintain a good workflow of midwives. Some key issues reported my midwives are, a) the software do not provide a notice if wrong information is added, b) the software systems do not let midwives know that the blood pressure is high. For example, one midwife (MW8) mentioned, "the other program [name of the software program] gives me some jokes. If I type 11 children it gives me a joke. If the baby's blood type is $O$ negative and we have to give medication. The software systems do not provide much intelligence. The whole thinking part is from the midwife. Though I believe that it cannot be fully taken over by the computer. The person who enters the data needs to take the decision".

Furthermore, the questionnaire data for prenatal registration collected from the pregnant women stays at the midwifery practice during the 9 months, when a baby is born and care has been finished, it goes to the national database anonymously [11]. For example, when someone has a miscarriage, midwife has to fill in a special form with all the information: there is no option in the software systems to do the same task. Midwives also use some point of care diagnostic devices such as blood pressure machine, Doppler machine, ultrasound in some practices and then sterilizing instruments are used for home delivery. These point-of-care (POC) testing devices are usually independent and each measurement data needs to enter into the software systems of the midwives.

Prenatal Visits and Quality of Care. The quality of care depends on the attitudes of the pregnant women and midwives. Sometimes some pregnant women have difficult behaviour. Some pregnant women do not even come for a follow up visit and they do not even keep their appointments up to date. One midwife mentioned (MW10), "though we have a booklet for the pregnant women and we note down their follow up appointments, still they miss the appointments". In the regions where low social status people do not come to a prenatal visits regularly. Midwives need a lot of interaction with the pregnant women. Sometimes people do not show up and midwives have to call them. It would be nice to have a reminder system for those pregnant women. One midwife mentioned, "the main part of our work is the relation with the pregnant women. If you do not have a good relation, you cannot give a good care. We do a lot of talking and listening. One of the basic competencies of midwives is making and building relations, maintaining relations and finishing the relations".

Barriers and Facilitators to Prenatal Care and IT. Four software systems are used in the midwifery centres such as a) MicroNatal (http://www.micronatal.nl/), b) Orfeus (http://www.orfeus.nl/), c) Onatal (http://www.onatal.nl/), and d) Vrumun (http://www.vrumun.nl/) and each midwifery centre uses only one of them. These software systems provide a wide range of support and generally the software system are secured and require secure log in credentials. One midwife (MW5) mentioned, "we have a system everybody has different discipline, I do not know the finance section of the software so I cannot access that part. You get a user name and a special 
password to $\log$ on to the system". The positive aspect is that the software allows midwives to provide direct feedback to the developers and helps to provide better care to midwives by giving overview of the finished tasks. However, midwives had mixed reaction about the current software systems. One of the key issues is the broken link among the different software systems of midwives and gynaecologists. One midwife mentioned, "I have my software and the gynaecologists have their own software. However, due to privacy reasons those are not connected. I want to check my patient within the system of the gynaecologists who is in a hospital".

Other wish list from the midwives were a) a touch program to input data while visiting a patient, b) easy customization of the menu items and other functional features where more frequent menus to be appeared only. One midwife mentioned, "when I have to visit a patient who cannot come to our midwifery centre, I use a diary to keep notes and patient data. I cannot access the patient data which are in my computer at the midwifery centre. It is a pity and I have to enter the notes and other information when I am back at my office”.

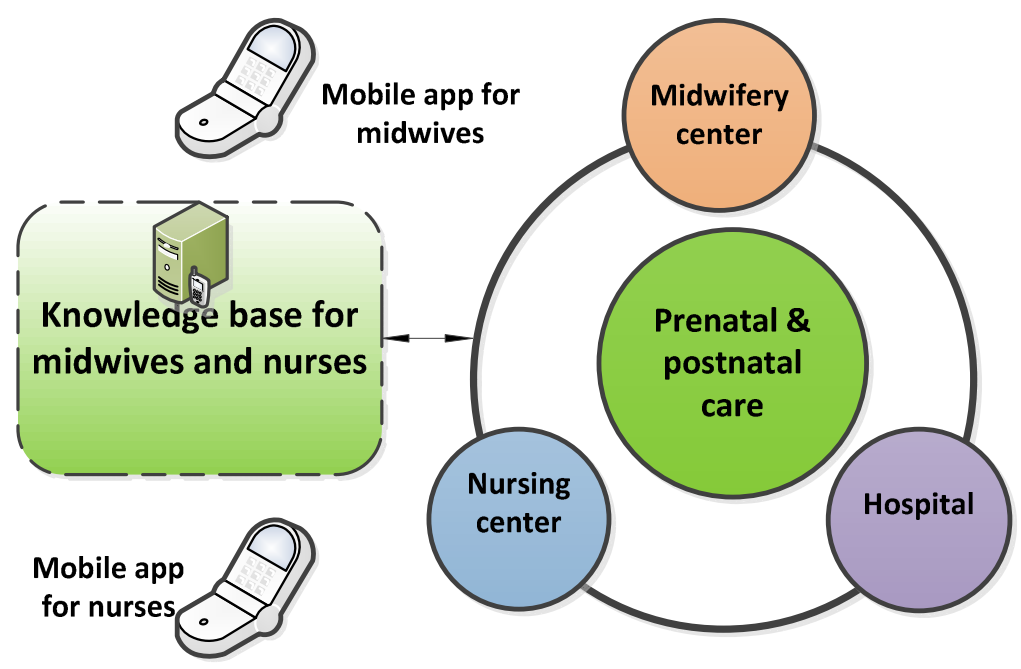

Fig. 1. Our proposed conceptual model to support improved prenatal and postnatal care. The keys issue is to repair the broken link among three care providers that is create an access to the patient data from each care location when required. Furthermore, mobile apps are proposed for midwives and nurses when they need to visit pregnant women and need to access the patient data. An integrated knowledge base is also proposed for improved care.

Midwives work closely with nurses (in Dutch they are called 'kraamzorg') and the nurses very often need critical information from midwives or specialists. For example, if a baby is born with high bilirubin which needs to be checked and very often nurses call midwives or specialists to know whether the bilirubin level is within normal range. One midwife mentioned (MW12), "this is really critical information and an assistive mobile application would certainly help the nurses and midwives to take a decision and reduces their burden". 


\section{Recommendations and Design Implications}

In this following section we provide recommendations to help midwives to provide better prenatal care.

Interoperability and Centralization of Software for Midwives. Too much information is provided to the pregnant women, therefore pregnant women either forget or they ask midwives repeatedly in upcoming visits. Therefore, it would be helpful if that information could be shared for both pregnant woman and midwives in an easy and accessible manner. For example, an interactive application/mobile app where it would be easy to interact with and pregnant women will get support over the nine months and post natal care would be really helpful.

It would be beneficial to have an integrated software system with doctors, midwives and other caregivers. The current system in the Netherlands does not allow different stakeholders to access the same patient data from their own software terminals. Therefore, creating an integrated health care system is a crucial need where privacy of the end user data will be preserved. The short term solution would be to make a viable link with the existing software systems operating in the midwifery centres and hospitals. We have observed that some of the software systems are not possible to use if the midwives need to visit a patient since the application can only be used at the computers of the midwifery centres. Therefore, a mobile application which could help to integrate with the local software system would be really helpful for the midwives. We recommend having a mobile app that would help to access the software systems while midwives have to visit a patient. The mobile app should be able to synchronise the gathered data later on.

Accessible Protocols. Since the maternal care system is based on protocols, and the obvious advantage would be to create some means to access the protocols based on the specific situation of pregnant women. Furthermore, creating a central knowledge base for the midwives would be helpful to reduce the burden for the new midwives.

Usability and Features of the Software for Midwives. We have found that there are four kinds of software systems and they have positive aspects while providing care by the midwives. All the software provides reasonable support to midwives despite some usability issues. The adoption process is based on price, and recommendations from other colleagues and the associated midwifery centres who work together. Improving the usability of the current midwifery software systems used in the midwifery centres will certainly help the workflow of the midwives. The major problems that we encountered were divided in two sides: a) lack of usable software system, and b) lack of financial support of the midwifery centres. One of the software was developed by individual initiative and no formal usability was measured. Furthermore, some manufacturers of the software systems started to get feedback from the midwives however since the community who use the systems are not very large the investment that is required to improve usability is less than the predicted return. We would like to stress that opinion of midwives should be taken into account while developing the software and support from the government is required for the software development firms. 
Virtual Midwives. Two types of user groups were identified who ask care from the midwives. One group is motivated to visit the midwifery centre and the other group often misses their appointments. We also found that midwives find their task harder due to attitude of the people which is hard to control. However, it would be helpful if midwife could be virtually connected with the patients which could reduce their workload. Most of the time midwives are overloaded with so many queries of the women who are first time pregnant. Therefore, creating a virtual community and getting regular reminders about appointments could help to bridge the situation. It has also been observed that some user groups are very motivated and eager to know the growth of their expected baby. Since the midwives check the growth once in a month and sometimes it is hard to follow some appointments it would be beneficial to have a software system that will inform pregnant women about the growth of their baby. Furthermore, the will help pregnant women to get a piece of mind and will lower the burden from the midwives for example they will receive less worrying question from the pregnant women. It is evident from existing literature that the workload for primary care midwives are huge [17].

Training for Midwives. Providing regular training to midwives about the usage of IT or existing software systems will improve their workflow. We observed that some midwives are not well aware about the capability of the IT systems. Therefore, regular training to make them competent will result in delivering improved care. These findings are also in line with the existing findings mentioned in [5, 8].

Tools to Support Postnatal Care. One of the issues midwives mentioned that they have problems in postnatal care support as well. The main reason was that the nurses involved in postnatal care are not sure about different measurements such as high bilirubin of the new born child. Most of the time the nurses call the midwives or doctors to consult. In some cases they have to calculate the bilirubin in every two days and calling nurses or doctors is very cumbersome. Therefore, a mobile application where nurses could measure the level of severity of the bilirubin would help to improve their workflow.

\section{Conclusion and Future Work}

In this paper we have presented a qualitative study to understand the work process and tools used to provide maternity care by midwives. The findings are encouraging though the prenatal care in the Netherlands is considered very good and some of the findings are due to the nature of the Dutch healthcare system. We are working with local midwives to design improved services for midwives and pregnant mothers. For example, we are designing an app for the peace of mind of pregnant mothers. The objective is to help pregnant mothers to get useful information and make them aware about the growth of the baby over 9 months. The app will also be able to document each visits to the midwifery centres and will provide recommendations on food and nutrition, exercise etc. Moreover, we believe that in order to bridge the different care providers, privacy of the information was an issue. KNOV is working to solve the issues so that each stakeholder can access each other's data to provide better pre and 
post natal care. In this study, we have interviewed the midwives from 12 different midwifery practices in the Netherlands. The experiences of the midwives varied in terms of software use, prior familiarity with IT and training. However, the findings led us to discover a better information system to improve maternal care in the Netherlands which may be suitable in similar other countries as well.

Acknowledgments. We thank the midwives and KNOV for their cooperation.

\section{References}

1. Anthony, S., Amelink-Verburg, M.P., Jacobusse, G.W., van der Pal-de Bruin, K.M.: De thuisbevalling in Nederland 1995-2002 (Home birth in the Netherlands 1995-2002) Bilthoven/Leiden, Stichting Perinatale Registratie Nederland, TNO Kwaliteit van Leven (2005)

2. Bais, J.M.J., Pel, M.: The basis of the Dutch obstetric system: risk selection. European Clinics in Obstetrics and Gynaecology 2(4), 209-212 (2006)

3. Bullough, C., Meda, N., et al.: Review: Current strategies for the reduction of maternal mortality. BJOG: An International Journal of Obstetrics \& Gynaecology 112(9), 11801188 (2005)

4. Dutch midwifery association, http: / /www. knov.nl

5. Gerrish, K., Morgan, L., et al.: Factors influencing use of information technology by nurses and midwives. Practice Development in Health Care 5(2), 92-101 (2006)

6. Havukumpu, J., Vähäkangas, P., Grönroos, E., Häkkinen, J.: Midwives experiences of using HMD in ultrasound scan. In: Proceedings of the 4th Nordic Conference on HumanComputer Interaction: Changing Roles, NordiCHI 2006, pp. 369-372. ACM (2006)

7. Herz, B.K., Measham, A.R.: The safe motherhood initiative: Proposals for action, World Bank (1987)

8. Hillan, E.M., McGuire, M.M., et al.: Computers in midwifery practice: a view from the labour ward. Journal of Advanced Nursing 27(1), 24-29 (2001)

9. McKenna, L., McLelland, G.: Midwives' use of the Internet: an Australian study. Midwifery 27(1), 74-79 (2011)

10. Manniën, J., Klomp, T., et al.: Evaluation of primary care midwifery in the Netherlands: design and rationale of a dynamic cohort study (DELIVER). BMC Health Services Research 12(1), 69 (2012)

11. Netherlands Perinatal Registry (PRN foundation), http://www . perinatreg.nl

12. Rooney, C.: Antenatal care and maternal health: how effective is it? A review of the evidence Maternal Health and safe Motherhood Programme division of Family Heath. WHO, Geneva (1992)

13. Smeenk, A.D.J., ten Have, H.A.M.J.: Medicalization and obstetric care: an analysis of developments in Dutch midwifery. Medicine, Health Care and Philosophy 6(2), 153-165 (2003)

14. Strauss, A., Corbin, J.M.: Basics of qualitative research: grounded theory procedures and techniques. Sage Publications (1990)

15. The state of world's midwifery, http://www. unfpa.org/sowmy/resources/en/main.htm

16. Wiegers, T.A., van der Zee, J., et al.: Variation in home birth rates between midwifery practices in the Netherlands. Midwifery 16(2), 96-104 (2000)

17. Wiegers T.A: Workload of primary-care midwives. Midwifery 23, 425-432 (2007); 7. NVOG: Nota 